\title{
Photosynthesis of a Dihydroimidazopyridine Chelate Shines Light on the Reactions of a Photoactivated Iron(III) Complex with $\mathrm{O}_{2}$
}

\author{
Christina Wegeberg, ${ }^{\dagger, \phi}$ Adiran de Aguirre, ${ }^{\not, \neq}$ Feliu Maseras $^{\natural}$ and Christine J. McKenzie ${ }^{\dagger, *}$ \\ + Department of Physics, Chemistry and Pharmacy, University of Southern Denmark, Campusvej \\ 55, 5230 Odense M (Denmark).*E-mail: mckenzie@sdu.dk \\ $\not$ Institute of Chemical Research of Catalonia (ICIQ), The Barcelona Institute of Science and \\ Technology, Avgda. Països Catalans, 16, 43007 Tarragona, Catalonia (Spain) \\ $\phi$ Present address: Department of Chemistry, University of Basel, St. Johanns-Ring 19, 4056 Basel \\ (Switzerland)
}

‡ Present address: University of Geneva, Department of Organic Chemistry, Quai Ernest Ansermet 30, 1211 Geneva 4 (Switzerland)

\section{Extended crystallographic description}

Table S1

Details of structure determination for $\left[\mathrm{Fe}_{2} \mathrm{O}\left(\mathrm{L}^{+}\right)_{2}\right]\left(\mathrm{ClO}_{4}\right)_{4}(\mathrm{MeCN})_{2}$

Table S2

Selected interatomic distances and angles in $\left[\mathrm{Fe}_{2} \mathrm{O}\left(\mathrm{L}^{+}\right)_{2}\right]\left(\mathrm{ClO}_{4}\right)_{4}(\mathrm{MeCN})_{2}$

Table S3

Selected experimental and calculated bonds and angles for $\left[\mathrm{Fe}_{2} \mathrm{O}(\mathrm{Cl})_{2}\left(\mathrm{~L}^{+}\right)_{2}\right]^{4+}$

Details of structure determination for $[\mathrm{Fe}(\mathrm{N}-\mathrm{NCS})(\mathrm{SBPy} 3)] \mathrm{ClO}_{4}$

Selected interatomic distances and angles in $\left[\mathrm{Fe}(\mathrm{N}-\mathrm{NCS})(\mathrm{SBPy} 3) \mathrm{ClO}_{4}\right.$

\section{Figure $\mathbf{S 1}$}

Table S6

Figure S2

NMR spectrum (d3-MeCN) of [ $\mathrm{Fe}(\mathrm{N}-\mathrm{NCS})(\mathrm{SBPy} 3)]^{+}$

Experimental and calculated selected distances for [Fe"(N-NCS)(SBPy3) $]^{+}$

Illustrations of the cation [Fe(OIPh)(tpena) $]^{+}$

Figure S3 Relative energies for 6- and 7-coordinated Fe-thiocyanto-tpena complexes S9

Table S7 TD-DFT results on absorption bands for the mer 7-coordinated isomers in sextet spin state

Table S8 TD-DFT results on absorption bands for the mer and fac 6-coordinated isomers in sextet spin state

Figure 54 Picture of solutions of $\left[\mathrm{Fe}(\mathrm{SCN})(\text { tpena) }]^{+}\right.$and its corresponding light-product

Figure S5

Relative free energies for $\left[\mathrm{Fe}\left(\mathrm{L}_{\text {open }}{ }^{\bullet}\right)(\mathrm{MeCN})_{\mathrm{x}}\right]^{2+}$ and $\left[\mathrm{Fe}\left(\mathrm{L}_{\text {closed }}{ }^{\bullet}\right)(\mathrm{MeCN})_{\mathrm{x}}\right]^{2+}(\mathrm{x}=0,1,2)$ $(x=0,1,2)$

Figure $\mathbf{S 6}$ Spin density distribution of $\left[\mathrm{Fe}\left(\mathrm{L}_{\text {closed }}{ }^{\bullet}\right)(\mathrm{MeCN})_{2}\right]^{2+}$

Figure S7 Relative energies for possible structures upon irradiation and $\mathrm{CO}_{2}$ release of $[\mathrm{Fe}(\mathrm{S}$ $\mathrm{SCN})(\text { tpena) }]^{+}$

Table S10 TD-DFT results on absorption bands for the possible intermediatiens upon irradiation of $[\mathrm{Fe}(\mathrm{S}-\mathrm{SCN})(\text { tpena })]^{+}$ 


\section{Single Crystal X-ray Diffraction Experiments}

The crystal structures of $\left[\mathrm{Fe}_{2} \mathrm{O}\left(\mathrm{L}^{+}\right)_{2}\right]\left(\mathrm{ClO}_{4}\right)_{4}(\mathrm{MeCN})_{2}$ ( $\mathrm{L}^{+}=2$-(2-(bis(pyridin-2-ylmethyl)amino)ethyl)2,3-dihydro-1H-imidazo[1,5-a]pyridin-4-iu) and [Fe(NCS)(SBPy3)]ClO 4 were collected at $100(2) \mathrm{K}$ on a Synergy, Dualflex, AtlasS2 diffractometer using CuK $\alpha$ radiation $(\lambda=1.54184 \AA$ ) and the CrysAlis PRO 1.171.39.12b suite ${ }^{1}$. Using SHELXLE ${ }^{2}$ the structures were solved by dual space methods $\left(\mathrm{SHELXT}^{3}\right.$ ) and refined on $\mathrm{F}^{2}$ using all the reflections (SHELXL-2018/34). All the non-hydrogen atoms were refined using anisotropic atomic displacement parameters, and the hydrogen atoms were inserted at calculated positions using a riding model. Crystal data, data collection and structure refinement details are summarized in Table S1 and S4. Selected bond distances and bond angles are summarized in Table S2 and S5.

\section{$\left.\mathrm{Fe}_{2} \mathrm{O}\left(\mathrm{L}^{+}\right)_{2}\right]\left(\mathrm{ClO}_{4}\right)_{4}(\mathrm{MeCN})_{2}$}

Based on bond distances and thermal ellipsoids, it was not possible to distinguish the position of the pyridine nitrogen in the five membered ring, neither is there a chemical reason for placing a nitrogen rather than a carbon in any of the two positions, hence N5 and C16 are treated as distorted over the two positions with 50:50 occupancy. Additional N5 and C16 were placed at same positions and with the same anisotropic displacement parameters. One of the perchlorate ions ( $\mathrm{Cl} 3 \mathrm{O}-\mathrm{O}-\mathrm{O} 9)$ is distorted; 07-09 was modelled over two positions with 80:20 occupancy. 07'-09' could not be modelled with anisotropic displacement parameters, because the atoms become non-positive definite atoms. The remaining electrons density is located around the distorted perchlorate ion but including distortion over three positions did not improve the model.

1. Rigaku Oxford Diffraction, (2018), CrysAlisPro Software system, version 1.171.39.27b, Rigaku Corporation, Oxford, UK.

2. C.B. Hübschle, G.M. Sheldrick and B. Dittrich. J. Appl. Cryst., 2011, 44, 1281-1284.

3. G.M. Sheldrick, Acta Cryst., 2015, A71, 3-8.

4. G.M. Sheldrick, Acta Cryst., 2015, C71, 3-8. 
Table S1. Crystal data for $\left[\mathrm{Fe}_{2} \mathrm{O}\left(\mathrm{L}^{+}\right)_{2}\right]\left(\mathrm{ClO}_{4}\right)_{4}(\mathrm{MeCN})_{2}$

\begin{tabular}{|c|c|}
\hline Chemical formula & $\mathrm{C}_{42} \mathrm{H}_{48} \mathrm{Fe}_{2} \mathrm{~N}_{10} \mathrm{Cl}{ }_{2} \mathrm{O} \cdot\left(\mathrm{ClO}_{4}\right)_{4} \cdot\left(\mathrm{C}_{2} \mathrm{H}_{3} \mathrm{~N}\right)_{2}$ \\
\hline$M_{\mathrm{r}}[\mathrm{g} / \mathrm{mol}]$ & 685.70 \\
\hline Crystal system & Monoclinic \\
\hline Space group & $P 2_{1} / n$ \\
\hline Temperature $[\mathrm{K}]$ & 100 \\
\hline$a, b, c[\AA]$ & $10.8036(1), 18.1231(2), 14.2869(1)$ \\
\hline$\beta\left[^{\circ}\right]$ & $97.789(1)$ \\
\hline$V\left[\AA^{3}\right]$ & $2771.49(4)$ \\
\hline$Z$ & 4 \\
\hline Radiation type & $\mathrm{Cu} K \alpha$ \\
\hline$\mu\left[\mathrm{mm}^{-1}\right]$ & 7.57 \\
\hline Crystal size $[\mathrm{mm}]$ & $0.18 \times 0.08 \times 0.06$ \\
\hline Colour & Orange \\
\hline Habit & Plate \\
\hline Diffractometer & $\begin{array}{l}\text { XtaLAB Synergy, Dualflex, AtlasS2 } \\
\text { diffractometer }\end{array}$ \\
\hline$T_{\min }, T_{\max }$ & $0.562,1.000$ \\
\hline Measured reflections & 64229 \\
\hline Independent reflections, $R_{\text {int }}$ & $5797,0.040$ \\
\hline Observed reflections $[I>2 \sigma(I)]$ & 5546 \\
\hline$(\sin \theta / \lambda)_{\max }\left(\AA^{-1}\right)$ & 0.631 \\
\hline$R\left[F^{2}>2 \sigma\left(F^{2}\right)\right]$ & 0.045 \\
\hline$w R\left(F^{2}\right), S$ & $0.167,1.50$ \\
\hline Reflection/parameters/restraints & $5797 / 389 / 511$ \\
\hline $\mathrm{H}$-atom treatment & H-atom parameters constrained \\
\hline Largest diff. peak and hole [e $\AA^{-3}$ ] & 1.74 and -0.78 \\
\hline
\end{tabular}

Computer programs: CrysAlis PRO 1.171.39.27b (Rigaku OD, 2015), SHELXL2014/5 (Sheldrick, 2014), SHELXL2014/6 (Sheldrick, 2014), Mercury 3.9 (Macrae at al., 2008) and SHELXL2014 (Sheldrick, 2014).

Table S2. Selected interatomic distances $[\AA ̊]$ and angles $\left[^{\circ}\right]$ in $\left[\mathrm{Fe}_{2} \mathrm{O}\left(\mathrm{L}^{+}\right)_{2}\right]\left(\mathrm{ClO}_{4}\right)_{4}(\mathrm{MeCN})_{2}$

\begin{tabular}{|l|l|}
\hline Fe1-O1 & $1.8053(4)$ \\
\hline Fe1-N1 & $2.113(2)$ \\
\hline Fe1-N3 & $2.132(2)$ \\
\hline Fe1-N2 & $2.171(2)$ \\
\hline Fe1-Cl1 & $2.2908(6)$ \\
\hline O1-Fe1 & $1.8053(4)$ \\
\hline
\end{tabular}

\begin{tabular}{|l|l|}
\hline O1-Fe1-N1 & $89.10(6)$ \\
\hline O1-Fe1-N3 & $95.19(6)$ \\
\hline N1-Fe1-N3 & $155.63(8)$ \\
\hline O1-Fe1-N2 & $91.48(5)$ \\
\hline N1-Fe1-N2 & $79.56(8)$ \\
\hline N3-Fe1-N2 & $76.35(8)$ \\
\hline O1-Fe1-Cl1 & $106.06(2)$ \\
\hline N1-Fe1-Cl1 & $103.53(6)$ \\
\hline N3-Fe1-Cl1 & $98.31(6)$ \\
\hline N2-Fe1-Cl1 & $162.15(6)$ \\
\hline Fe1-O1-Fe1 & 180.0 \\
\hline
\end{tabular}

iSymmetry code: (i) $-x,-y+1,-z+1$. 


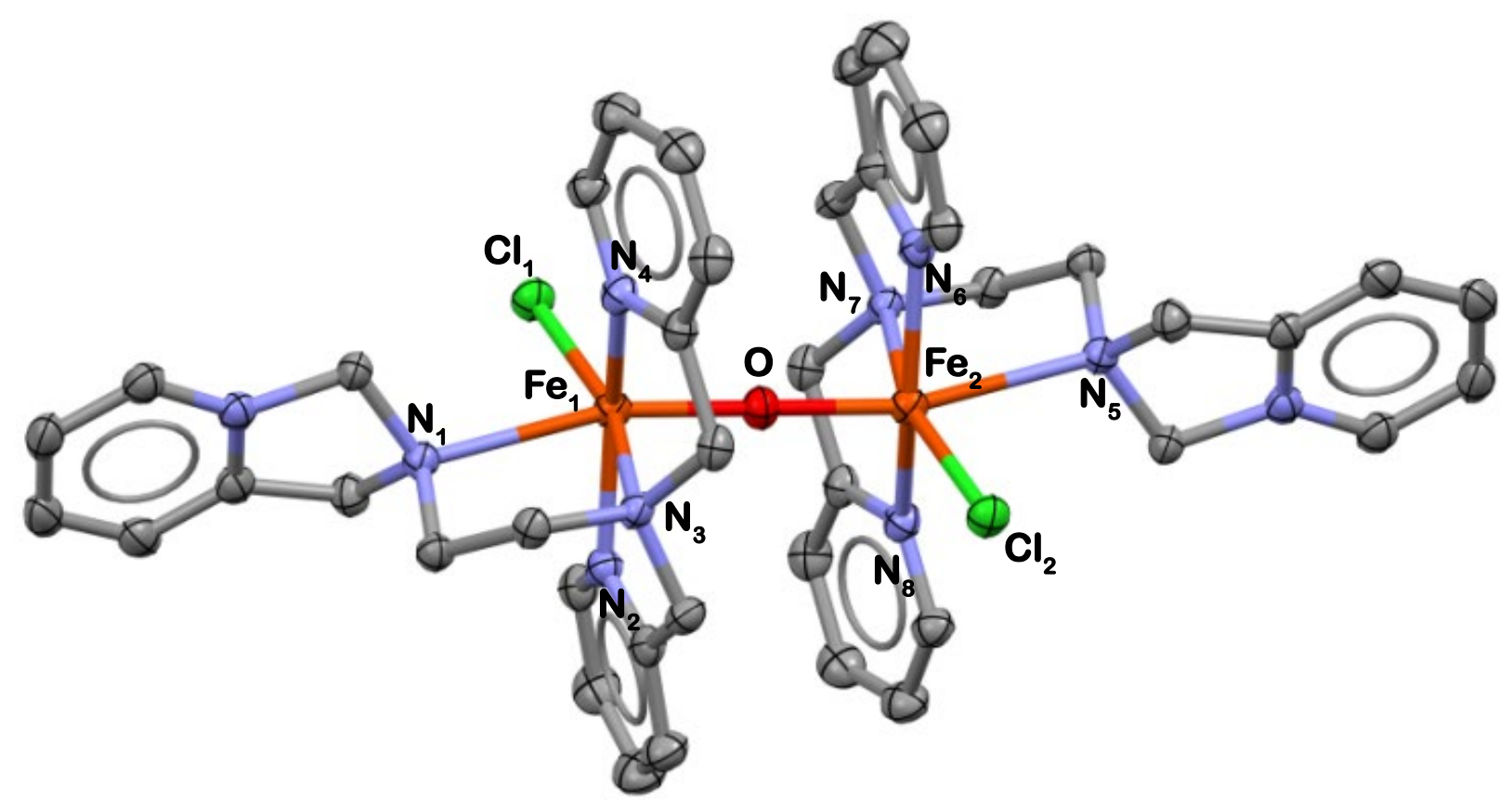

Table S3. Comparison between experimental and optimized selected bonds and angles for $\left[\mathrm{Fe}_{2} \mathrm{O}(\mathrm{Cl})_{2}\left(\mathrm{~L}^{+}\right)_{2}\right]^{4+}$. Distances are in $\AA$ and angles in .

\begin{tabular}{|c|c|c|c|c|c|c|c|c|}
\hline & $\begin{array}{l}\mathrm{Fe}_{1}-\mathrm{N}_{1} \\
\mathrm{Fe}_{2}-\mathrm{N}_{5}\end{array}$ & $\begin{array}{l}F e_{1}-N_{2} \\
F e_{2}-N_{6}\end{array}$ & $\begin{array}{l}\mathrm{Fe}_{1}-\mathrm{N}_{3} \\
\mathrm{Fe}_{2}-\mathrm{N}_{6}\end{array}$ & $\begin{array}{l}\mathrm{Fe}_{1}-\mathrm{N}_{4} \\
\mathrm{Fe}_{2}-\mathrm{N}_{8}\end{array}$ & $\begin{array}{l}\mathrm{Fe}_{1}-\mathrm{O} \\
\mathrm{Fe}_{2}-\mathrm{O}\end{array}$ & $\begin{array}{l}\mathrm{Fe}_{1}-\mathrm{Cl}_{1} \\
\mathrm{Fe}_{2}-\mathrm{Cl}_{2}\end{array}$ & $\mathrm{Fe}_{1-}$ & $\begin{array}{l}N_{1}-\mathrm{Fe}_{1}-\mathrm{O} \\
\mathrm{N}_{5}-\mathrm{Fe}_{2}-\mathrm{O}\end{array}$ \\
\hline Exp. & 2.46 & 2.11 & 2.17 & 2.13 & 1.81 & 2.29 & 180.0 & 165.3 \\
\hline$S=0$ & $21,2.21$ & $98,2.04$ & $.00,2.00$ & $04,1.97$ & $.75,1.75$ & $32,2.33$ & 161.3 & $175.6,177.0$ \\
\hline$S=1$ & 2.1 & 1. & 00 & & & .35 & .9 & 5.2 \\
\hline$S=2$ & $2.54,2.17$ & $1.98,2.01$ & $2.11,2.00$ & & $1.78,1.80$ & $2.41,2.32$ & 173.5 & 171. \\
\hline$S=3$ & $2.55,2.23$ & $1.99,2.12$ & $2.08,2.24$ & $2.02,2.06$ & $1.77,1.80$ & $2.35,2.39$ & 173.4 & $169.4,171.0$ \\
\hline$S=4$ & $2.44,2.22$ & $2.14,2.12$ & $2.22,2.26$ & $2.17,2.06$ & $1.78,1.78$ & $2.34,2.38$ & 173.5 & 169.5 \\
\hline$S=5$ & $2.41,2.41$ & $2.14,2.16$ & $2.22,2.22$ & $2.16,2.14$ & $1.82,1.82$ & $2.33,2.33$ & 174.9 & $168.2,168.1$ \\
\hline
\end{tabular}


Table S4. Crystal data for $[\mathrm{Fe}(\mathrm{N}-\mathrm{NCS})(\mathrm{SBPy} 3)] \mathrm{ClO}_{4}$

\begin{tabular}{|c|c|}
\hline Chemical formula & $\mathrm{C}_{21} \mathrm{H}_{21} \mathrm{FeN}_{6} \mathrm{~S} \cdot \mathrm{ClO}_{4}$ \\
\hline$M_{\mathrm{r}}[\mathrm{g} / \mathrm{mol}]$ & 544.80 \\
\hline Crystal system & Triclinic \\
\hline Space group & $P^{-} 1$ \\
\hline Temperature [K] & 100 \\
\hline$a, b, c[\AA]$ & $10.9366(5), 13.2530(6), 16.3118(8)$ \\
\hline$\alpha, \beta, \gamma\left[^{\circ}\right]$ & $104.559(4), 92.332(4), 90.985(4)$ \\
\hline$V\left[\AA^{3}\right]$ & $2285.54(19)$ \\
\hline$Z$ & 4 \\
\hline Radiation type & $\mathrm{Cu} K \alpha$ \\
\hline$\mu\left[\mathrm{mm}^{-1}\right]$ & 7.59 \\
\hline Crystal size $[\mathrm{mm}]$ & $0.08 \times 0.04 \times 0.02$ \\
\hline Colour & Red \\
\hline Habit & Needle \\
\hline Diffractometer & $\begin{array}{l}\text { XtaLAB Synergy, Dualflex, AtlasS2 } \\
\text { diffractometer }\end{array}$ \\
\hline$T_{\min }, T_{\max }$ & $0.709,0.909$ \\
\hline Measured reflections & 210929 \\
\hline Independent reflections, $R_{\text {int }}$ & $9080,0.074$ \\
\hline Observed reflections $[I>2 \sigma(I)]$ & 7250 \\
\hline$(\sin \theta / \lambda)_{\max }\left(\AA^{-1}\right)$ & 0.628 \\
\hline$R\left[F^{2}>2 \sigma\left(F^{2}\right)\right]$ & 0.061 \\
\hline$w R\left(F^{2}\right), S$ & $0.168,1.04$ \\
\hline Reflection/parameters/restraints & $9080 / 613 / 0$ \\
\hline $\mathrm{H}$-atom treatment & H-atom parameters constrained \\
\hline Largest diff. peak and hole [e $\AA^{-3}$ ] & 1.50 and -1.27 \\
\hline
\end{tabular}

Computer programs: CrysAlis PRO 1.171.39.27b (Rigaku OD, 2015), SHELXL2014/5 (Sheldrick, 2014), SHELXL2014/6 (Sheldrick, 2014), Mercury 3.9 (Macrae at al., 2008) and SHELXL2014 (Sheldrick, 2014). 
Table S5. Selected interatomic distances $[\AA ̊]$ and angles $\left[{ }^{\circ}\right]$ in $[\mathrm{Fe}(\mathrm{N}-\mathrm{NCS})(\mathrm{SBPy} 3)] \mathrm{ClO}_{4}$

\begin{tabular}{|l|l|}
\hline Fe1A-N2A & $1.858(4)$ \\
\hline Fe1A-N1A & $1.947(4)$ \\
\hline Fe1A-N6A & $1.953(4)$ \\
\hline Fe1A-N5A & $1.974(3)$ \\
\hline Fe1A-N4A & $1.985(3)$ \\
\hline Fe1A-N3A & $1.988(4)$ \\
\hline & \\
\hline N2A-Fe1A-N1A & $81.98(16)$ \\
\hline N2A-Fe1A-N6A & $175.22(14)$ \\
\hline N1A-Fe1A-N6A & $97.33(15)$ \\
\hline N2A-Fe1A-N5A & $87.25(14)$ \\
\hline N1A-Fe1A-N5A & $96.93(15)$ \\
\hline N6A-Fe1A-N5A & $88.14(14)$ \\
\hline N2A-Fe1A-N4A & $94.12(14)$ \\
\hline N1A-Fe1A-N4A & $96.98(13)$ \\
\hline N6A-Fe1A-N4A & $90.66(14)$ \\
\hline N5A-Fe1A-N4A & $166.09(16)$ \\
\hline N2A-Fe1A-N3A & $86.81(17)$ \\
\hline N1A-Fe1A-N3A & $168.60(16)$ \\
\hline N6A-Fe1A-N3A & $94.01(16)$ \\
\hline N5A-Fe1A-N3A & $84.52(16)$ \\
\hline N4A-Fe1A-N3A & $81.74(14)$ \\
\hline
\end{tabular}

\begin{tabular}{|l|l|}
\hline Fe1B-N2B & $1.867(3)$ \\
\hline Fe1B-N1B & $1.934(3)$ \\
\hline Fe1B-N6B & $1.959(4)$ \\
\hline Fe1B-N5B & $1.969(3)$ \\
\hline Fe1B-N4B & $1.984(4)$ \\
\hline Fe1B-N3B & $1.988(3)$ \\
\hline & \\
\hline N2B-Fe1B-N1B & $82.07(14)$ \\
\hline N2B-Fe1B-N6B & $178.11(16)$ \\
\hline N1B-Fe1B-N6B & $97.23(14)$ \\
\hline N2B-Fe1B-N5B & $87.64(15)$ \\
\hline N1B-Fe1B-N5B & $99.46(14)$ \\
\hline N6B-Fe1B-N5B & $90.75(14)$ \\
\hline N2B-Fe1B-N4B & $94.24(14)$ \\
\hline N1B-Fe1B-N4B & $93.96(13)$ \\
\hline N6B-Fe1B-N4B & $87.56(14)$ \\
\hline N5B-Fe1B-N4B & $166.58(14)$ \\
\hline N2B-Fe1B-N3B & $86.90(15)$ \\
\hline N1B-Fe1B-N3B & $167.94(14)$ \\
\hline N6B-Fe1B-N3B & $93.95(14)$ \\
\hline N5B-Fe1B-N3B & $84.87(14)$ \\
\hline N4B-Fe1B-N3B & $81.97(14)$ \\
\hline
\end{tabular}




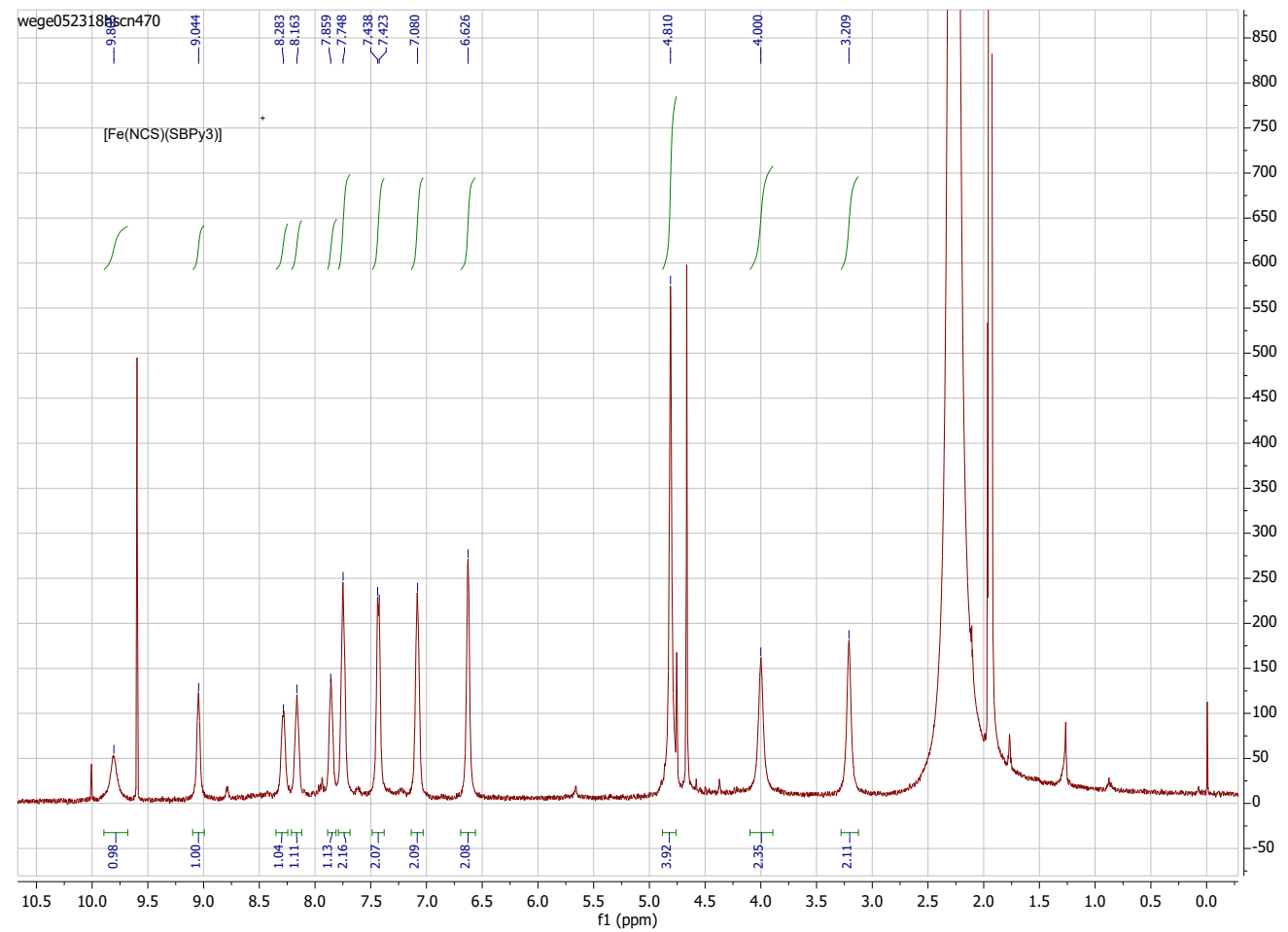

Figure S1. NMR spectrum of [Fe"(NCS)(SBPy3) $]^{+}$formed upon irradiation of $\left[\mathrm{Fe}(\mathrm{SCN})(\text { tpena) }]^{+}\right.$in $\mathrm{MeCN}-d_{3} . \quad \delta$ $\left.=9.81(1 \mathrm{H}, \mathrm{s}, \mathrm{N}=\mathrm{C} \underline{\mathrm{H}}), 9.05\left(1 \mathrm{H}, \mathrm{s}, \mathrm{Py}^{\prime}\right), 8.28(1 \mathrm{H}, \mathrm{d}, J=6.4 \mathrm{~Hz}, \mathrm{Py})^{\prime}\right), 8.16\left(1 \mathrm{H}, \mathrm{t}, J=8.4 \mathrm{~Hz}, \mathrm{Py}^{\prime}\right), 7.86\left(1 \mathrm{H}, \mathrm{s}, \mathrm{Py}{ }^{\prime}\right)$, $7.75\left(2 \mathrm{H}, \mathrm{t}, J=8.0 \mathrm{~Hz}, \mathrm{Py}{ }^{\prime \prime}\right), 7.42\left(2 \mathrm{H}, \mathrm{d}, J=6.4 \mathrm{~Hz}, \mathrm{Py}^{\prime \prime}\right), 7.08\left(2 \mathrm{H}, \mathrm{t}, J=6.0 \mathrm{~Hz}, \mathrm{Py}{ }^{\prime \prime}\right), 6.63(2 \mathrm{H}, \mathrm{d}, J=2.0 \mathrm{~Hz}$, $\left.\mathrm{Py}^{\prime \prime}\right), 4.81\left(4 \mathrm{H}, \mathrm{s}, \mathrm{C}_{2} \mathrm{Py}^{\prime \prime}\right), 4.00\left(2 \mathrm{H}, \mathrm{s}, \mathrm{NC}_{2}\right), 3.21\left(2 \mathrm{H}, \mathrm{s}, \mathrm{NC}_{2}\right) \mathrm{ppm}$. The singlet at $\delta=9.60$ originates from formaldehyde released as a product in the photo-triggered formation of [ $\mathrm{Fe}$ "I $(\mathrm{N}-\mathrm{NCS})(\mathrm{SBPy} 3)]^{+}$from [Fe(S$\mathrm{SCN})(\text { tpena) }]^{+}$.

Table S6. Experimental and calculated selected distances for [Fe"(N-NCS)(SBPy3)]+. Distances in Å. Energies in $\mathrm{kcal} / \mathrm{mol}$.

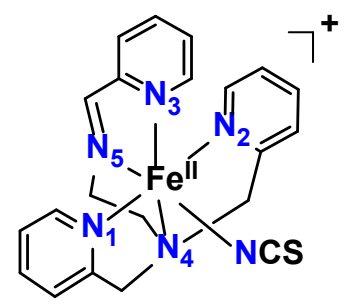

\begin{tabular}{|l|c|c|c|c|c|c|c|}
\hline & $\mathbf{d}($ Fe-NCS) & $\mathbf{d}($ Fe-N1) & $\mathbf{d}$ (Fe-N2) & $\mathbf{d}$ (Fe-N3) & $\mathbf{d}$ (Fe-N4) & d (Fe-N5) & $\begin{array}{c}\text { Free } \\
\text { Energy }\end{array}$ \\
\hline Experimental & 1.9533 & 1.9846 & 1.9740 & 1.9473 & 1.9886 & 1.8578 & -- \\
\hline Singlet (S=0) & 1.9593 & 1.9969 & 1.9865 & 1.9892 & 2.0126 & 1.8921 & 0.0 \\
\hline Triplet (S=1) & 1.9243 & 1.9904 & 1.9782 & 1.9672 & 2.0050 & 1.9104 & 24.9 \\
\hline Quintet (S=2) & 2.0677 & 2.2230 & 2.2160 & 2.2278 & 2.3091 & 2.1560 & -6.1 \\
\hline
\end{tabular}

The distances are in agreement with a diamagnetic (singlet spin state) complex consistent with NMR spectrum and X-ray crystal structure and we consider the computed energies sufficiently close to support the assignment. 


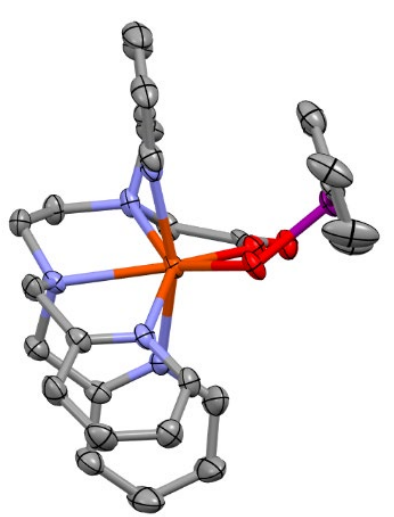

A

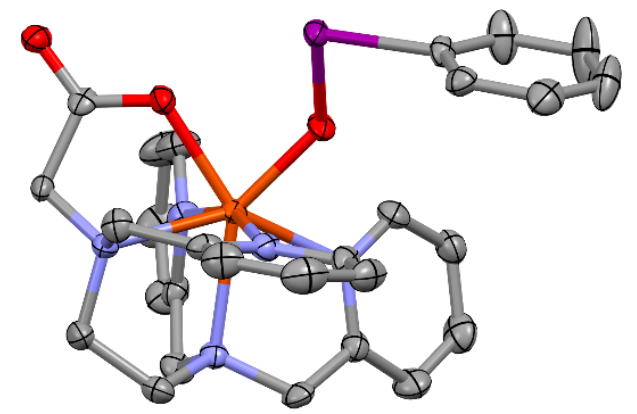

B

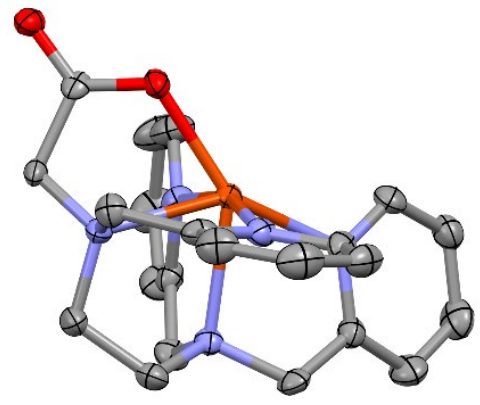

C

Figure S2. The cation $[\mathrm{Fe}(\mathrm{OIPh})(\text { tpena })]^{2+}(\mathrm{CCDC}$ reference code: TEFVUX) is seen from two different orientations in $\mathrm{A}$ and $\mathrm{B}$. In $\mathrm{C}$ the iodosylbenzene ligand is omitted to highlight that the ligand tpena is mainly covering one side of the iron center leaving room for coordination of a seventh donor. Hydrogen atoms are in all three presentations omitted for clarity. 

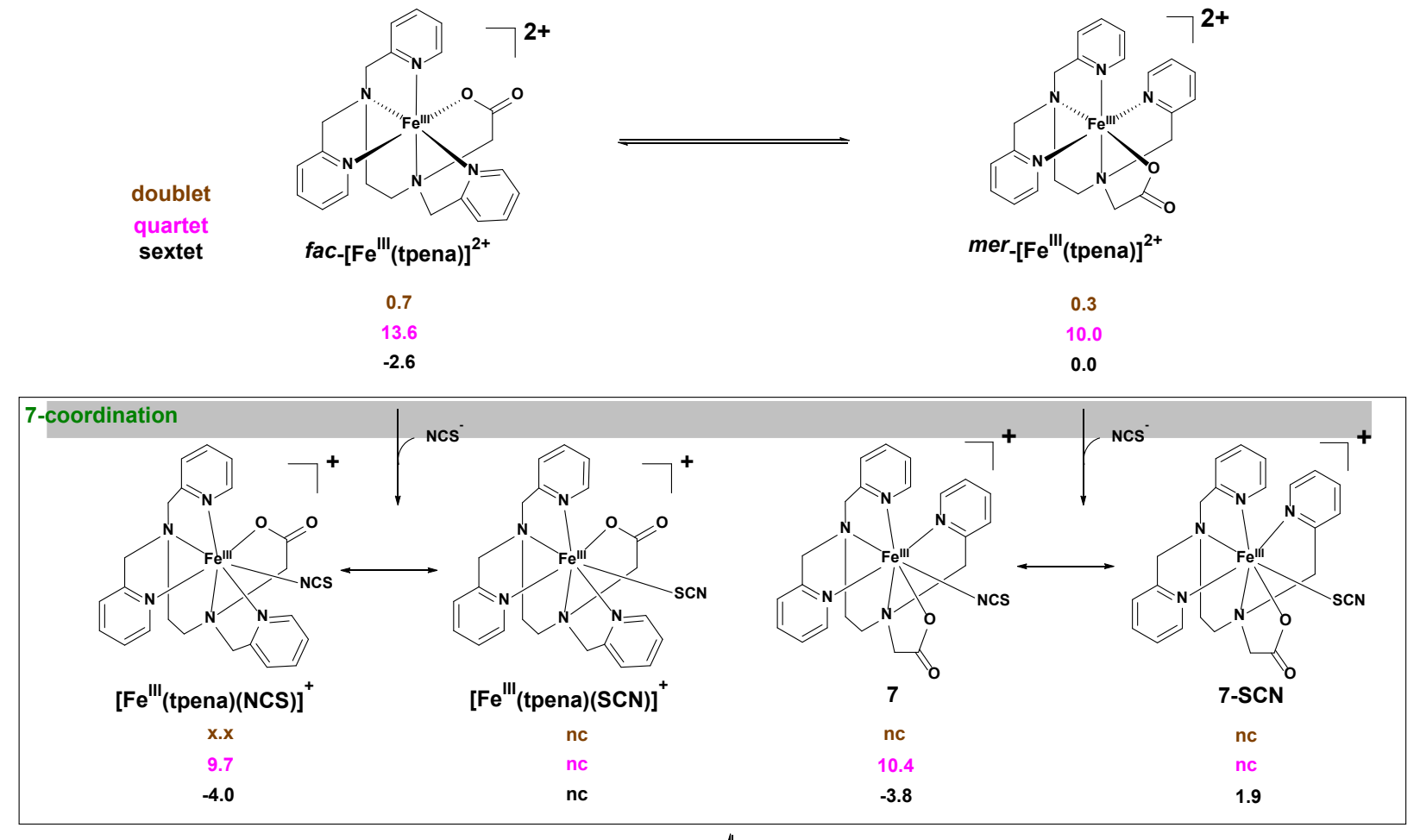

pyridine
decoordination

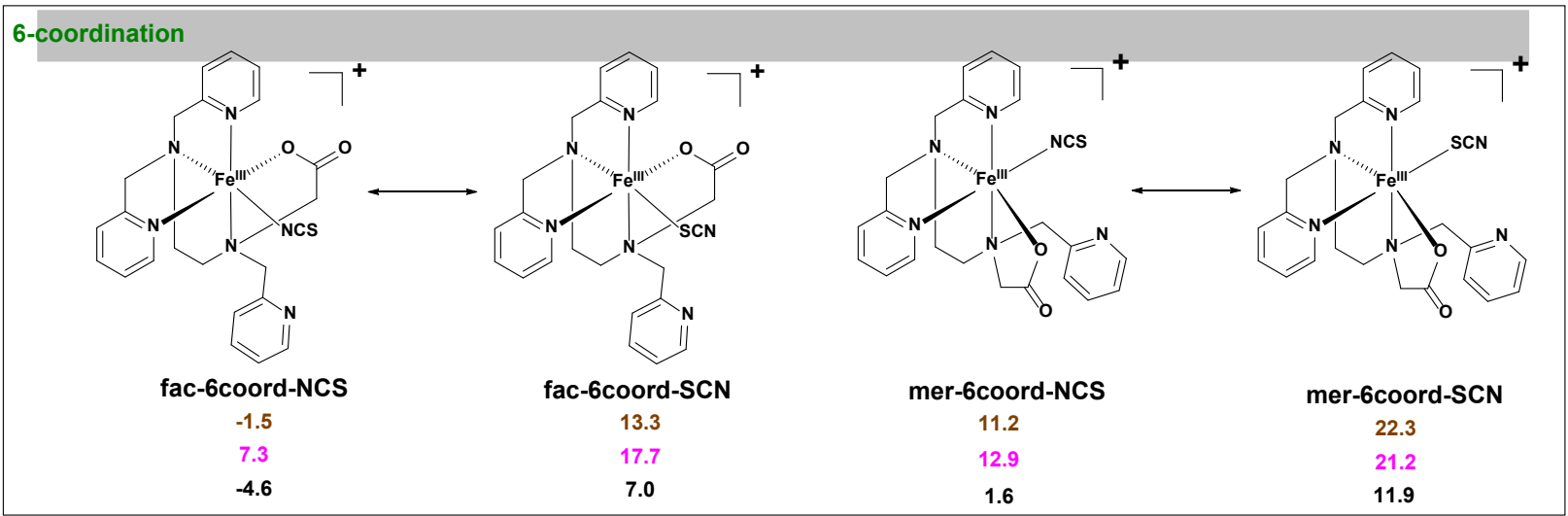

Figure S3. Structures accesible upon coordination of NCS or SCN. Relative energies are free energies in $\mathrm{kcal} / \mathrm{mol}$ from sextet spin state of the initial complex mer-[Fe"'(tpena) ${ }^{2+}$. Brown: doublet, purple: quartet, black: sextet. Structures marked as nc did not converge, or the optimization ended up to a 6-coordination isomer after decoordination of the NCS (or SCN) ligand or one of the pyiridnes arms of the tpena ligand. 
Table S7. TD-DFT results on absorption bands for the 7-coordinated isomers in their sextet spin state.

\begin{tabular}{|c|c|}
\hline $\begin{array}{l}\text { 7-SCN (S = 5/2) } \\
\text { Exc.Stat. 1: Energy = } 538 \mathrm{~nm}, \\
f=0.0308,122 B-124 B 0.63, \\
123 B-124 B-0.57 \\
\text { Exc.Stat. } 2: \text { Energy }=511 \mathrm{~nm}, \\
f=0.0029,122 B-124 B 0.57 \\
123 B-124 B 0.53 \\
\text { Exc.Stat. 3: Energy }=506 \mathrm{~nm}, \\
f=0.0010,122 B-125 B 0.72 \\
\text { Exc.Stat. 4: Energy }=476 \mathrm{~nm}, \\
f=0.0857,122 B-126 B 0.61 \\
123 B-126 B-0.50 \\
\text { Exc.Stat. 5: Energy }=431 \mathrm{~nm}, \\
f=0.0004,123 B-125 B 0.81 \\
\text { Exc.Stat. 6: Energy }=421 \mathrm{~nm}, \\
f=0.0876,123 B-126 B 0.68, \\
122 B-126 B 0.52 \\
\text { Exc.Stat. 7: Energy }=405 \mathrm{~nm}, \\
f=0.0112,122 B-127 B 0.84\end{array}$ & $\begin{array}{l}7 \text { (S = 5/2) } \\
\text { Exc.Stat. 1: Energy }=430 \mathrm{~nm}, \\
\mathrm{f}=0.0010,123 \mathrm{~B}-124 \mathrm{~B} 0.91 \\
\text { Exc.Stat. 2: Energy }=419 \mathrm{~nm}, \\
\mathrm{f}=0.0469,122 \mathrm{~B}-124 \mathrm{~B} 0.85 \\
\text { Exc.Stat. 3: Energy }=404 \mathrm{~nm}, \\
\mathrm{f}=0.0439,123 \mathrm{~B}-125 \mathrm{~B} 0.60 \\
\text { Exc.Stat. 4: Energy }=402 \mathrm{~nm}, \\
\mathrm{f}=0.0005,122 \mathrm{~A}-125 \mathrm{~A} 0.84 \\
\text { Exc.Stat. 5: Energy }=393 \mathrm{~nm}, \\
\mathrm{f}=0.0097,117 \mathrm{~B}-124 \mathrm{~B} 0.45 \\
\text { Exc.Stat. 6: Energy }=386 \mathrm{~nm}, \\
\mathrm{f}=0.0236,123 \mathrm{~B}-125 \mathrm{~B} 0.56\end{array}$ \\
\hline
\end{tabular}


Table S8. TD-DFT results on absorption bands for the mer and fac 6-coordinated isomers of iron-tpenathiocyanate complexes in their sextet spin state.

\begin{tabular}{|c|c|}
\hline mer-[6coord-NCS] ${ }^{+}(S=5 / 2)$ & mer-[6coord-SCN)]+ $(S=5 / 2)$ \\
\hline $\begin{array}{l}\text { Exc. Stat. 1: Energy }=458 \mathrm{~nm} \text {, } \\
f=0.0032,123 \mathrm{~B}-124 \mathrm{~B} 0.91\end{array}$ & $\begin{array}{l}\text { Exc.Stat. 1: Energy }=494 \mathrm{~nm}, \\
\mathrm{f}=0.0170,123 \mathrm{~B}-124 \mathrm{~B} 0.91\end{array}$ \\
\hline $\begin{array}{l}\text { Exc.Stat. 2: Energy }=445 \mathrm{~nm}, \\
f=0.0156,122 \mathrm{~B}-124 \mathrm{~B} 0.82\end{array}$ & $\begin{array}{l}\text { Exc.Stat. 2: Energy }=473 \mathrm{~nm}, \\
\mathrm{f}=0.0077,121 \mathrm{~B}-124 \mathrm{~B} 0.72\end{array}$ \\
\hline $\begin{array}{l}\text { Exc.Stat. 3: Energy }=425 \mathrm{~nm} \text {, } \\
\mathrm{f}=0.0042,122 \mathrm{~B}-125 \mathrm{~B} 0.69\end{array}$ & $\begin{array}{l}\text { Exc.Stat. 3: Energy }=461 \mathrm{~nm}, \\
\mathrm{f}=0.0021,123 \mathrm{~B}-125 \mathrm{~B} 0.66\end{array}$ \\
\hline $\begin{array}{l}\text { Exc.Stat. 4: Energy }=410 \mathrm{~nm} \text {, } \\
f=0.0017,120 \mathrm{~B}-124 \mathrm{~B} 0.53\end{array}$ & $\begin{array}{l}\text { Exc.Stat. 4: Energy }=449 \mathrm{~nm}, \\
\mathrm{f}=0.0067,123 \mathrm{~B}-126 \mathrm{~B} 0.66\end{array}$ \\
\hline $\begin{array}{l}\text { Exc.Stat. 5: Energy }=395 \mathrm{~nm}, \\
\mathrm{f}=0.1755,123 \mathrm{~B}-125 \mathrm{~B} 0.57\end{array}$ & $\begin{array}{l}\text { Exc.Stat. 5: Energy }=437 \mathrm{~nm}, \\
\mathrm{f}=0.0010,123 \mathrm{~B}-125 \mathrm{~B} 0.64\end{array}$ \\
\hline $\begin{array}{l}\text { Exc.Stat. 6: Energy }=383 \mathrm{~nm} \text {, } \\
f=0.0374,120 \mathrm{~B}-125 \mathrm{~B} 0.45 \\
\text { Exc.Stat. 7: Energy }=379 \mathrm{~nm}, \\
f=0.0326,120 \mathrm{~B}-126 \mathrm{~B} 0.43\end{array}$ & $\begin{array}{l}\text { Exc.Stat. 6: Energy }=416 \mathrm{~nm}, \\
\mathrm{f}=0.0152,121 \mathrm{~B}-126 \mathrm{~B} 0.57\end{array}$ \\
\hline fac-[6coord-NCS] ${ }^{+}(S=5 / 2)$ & $f a c-[6 \text { coord-SCN }]^{+}(\mathrm{S}=5 / 2)$ \\
\hline $\begin{array}{l}\text { Exc.Stat. 1: Energy }=447 \mathrm{~nm}, \\
\mathrm{f}=0.0001,123 \mathrm{~B}-124 \mathrm{~B} 0.95\end{array}$ & $\begin{array}{l}\text { Exc.Stat. 1: Energy }=453 \mathrm{~nm}, \\
\mathrm{f}=0.0262,123 \mathrm{~B}-124 \mathrm{~B} 0.91\end{array}$ \\
\hline $\begin{array}{l}\text { Exc. Stat. 2: Energy }=437 \mathrm{~nm} \text {, } \\
f=0.0178,122 \mathrm{~B}-124 \mathrm{~B} 0.80\end{array}$ & $\begin{array}{l}\text { Exc.Stat. 2: Energy }=438 \mathrm{~nm}, \\
\mathrm{f}=0.0021 .123 \mathrm{~B}-126 \mathrm{~B} 0.84\end{array}$ \\
\hline $\begin{array}{l}\text { Exc.Stat. 3: Energy }=416 \mathrm{~nm} \text {, } \\
f=0.0002 .122 B-125 B 0.94\end{array}$ & $\begin{array}{l}\text { Exc.Stat. 3: Energy }=423 \mathrm{~nm}, \\
\mathrm{f}=0.0004 .117 \mathrm{~B}-124 \mathrm{~B} 0.69\end{array}$ \\
\hline $\begin{array}{l}\text { Exc.Stat. 4: Energy }=385 \mathrm{~nm} \text {, } \\
\mathrm{f}=0.1460,123 \mathrm{~B}-125 \mathrm{~B} 0.66\end{array}$ & $\begin{array}{l}\text { Exc.Stat. 4: Energy }=416 \mathrm{~nm}, \\
\mathrm{f}=0.0000,123 \mathrm{~B}-125 \mathrm{~B} 0.85\end{array}$ \\
\hline $\begin{array}{l}\text { Exc.Stat. 5: Energy }=380 \mathrm{~nm} \text {, } \\
f=0.0672,122 \mathrm{~B}-126 \mathrm{~B} 0.63\end{array}$ & $\begin{array}{l}\text { Exc.Stat. 5: Energy }=393 \mathrm{~nm}, \\
\mathrm{f}=0.0080,117 \mathrm{~B}-126 \mathrm{~B} 0.65\end{array}$ \\
\hline $\begin{array}{l}\text { Exc.Stat. 6: Energy }=375 \mathrm{~nm} \text {, } \\
f=0.0230,114 \mathrm{~B}-124 \mathrm{~B} 0.44\end{array}$ & $\begin{array}{l}\text { Exc.Stat. 6: Energy }=383 \mathrm{~nm}, \\
\mathrm{f}=0.0172,119 \mathrm{~B}-124 \mathrm{~B} 0.39 \\
\text { Exc.Stat. } 7: \text { Energy }=379 \mathrm{~nm}, \\
f=0.0013,117 \mathrm{~B}-125 \mathrm{~B} 0.63\end{array}$ \\
\hline
\end{tabular}




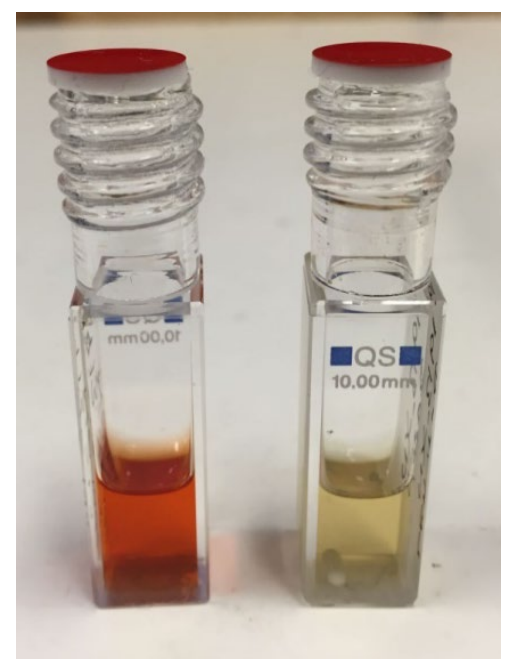

Figure S4. Picture of solutions of (left) $[\mathrm{Fe}(\mathrm{SCN}) \text { (tpena) }]^{+}$and (right) its corresponding light-product. 


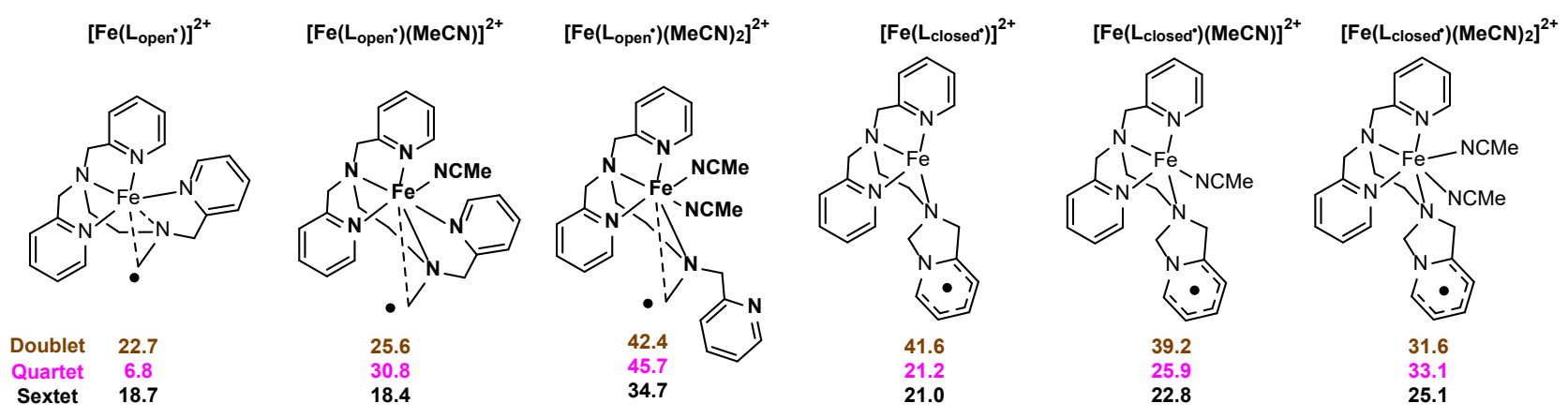

Figure S5. $\left[\mathrm{Fe}\left(\mathrm{L}_{\text {open }}{ }^{\bullet}\right)\right]^{2+}$ and its possible isomers considered in the Mössbauer parameter calculations. The energies in this figure are not conclusive, as the intermediate comes down from the excited state, and in principle has no limitations in terms of energy.

Table S9. DFT-calculated Mössbauer parameters: isomer shift $(\delta)$ and quadrupole splitting $\left(\Delta \mathrm{E}_{\mathrm{Q}}\right)$, for starting complexes, product and suggested transient species. Experimental values are provided for comparison.

\begin{tabular}{|c|c|c|c|c|c|}
\hline & \multirow[b]{2}{*}{$S_{\text {complex }}$} & \multicolumn{2}{|c|}{$\delta\left[\mathrm{mm} \mathrm{s}^{-1}\right]$} & \multicolumn{2}{|c|}{$\Delta \mathrm{E}_{\mathrm{Q}}\left[\mathrm{mm} \mathrm{s}^{-1}\right]$} \\
\hline & & Exp & calc & $\exp$ & Calc \\
\hline Experimental & $1 / 2$ & 0.45 & & singlet & \\
\hline \multirow{3}{*}[\mathrm{Fe}(\mathrm{L}_{\text{open}}{}^{\bullet})]{$^{2+}$} & $1 / 2$ & & 0.43 & & 1.42 \\
\hline & $3 / 2$ & & 0.79 & & 3.71 \\
\hline & $5 / 2$ & & 0.78 & & 2.89 \\
\hline \multirow{3}{*}[\mathrm{Fe}(\mathrm{L}_{\text{open}}{}^{\circ})(\mathrm{MeCN})]{$^{2+}$} & $1 / 2$ & & 0.64 & & 0.65 \\
\hline & $3 / 2$ & & 0.69 & & -1.46 \\
\hline & $5 / 2$ & & 0.91 & & 3.07 \\
\hline \multirow{3}{*}[\mathrm{Fe}(\mathrm{L}_{\text{open}}{}^{\circ})(\mathrm{MeCN})_{2}]{$^{2+}$} & $1 / 2$ & & 0.62 & & 0.79 \\
\hline & $3 / 2$ & & 0.63 & & 1.76 \\
\hline & $5 / 2$ & & 0.92 & & 3.01 \\
\hline \multirow{3}{*}[\mathrm{Fe}(\mathrm{L}_{\text{closed}}{}^{\circ})]{$^{2+}$} & $1 / 2$ & & 0.94 & & 1.88 \\
\hline & $3 / 2$ & & 1.04 & & 2.30 \\
\hline & $5 / 2$ & & 0.91 & & 1.56 \\
\hline \multirow{3}{*}[\mathrm{Fe}(\mathrm{L}_{\text{closed}}{}^{\circ})(\mathrm{MeCN})]{$^{2+}$} & $1 / 2$ & & 0.69 & & -1.96 \\
\hline & $3 / 2$ & & 1.08 & & -1.33 \\
\hline & $5 / 2$ & & 0.87 & & 2.98 \\
\hline \multirow{3}{*}[\mathrm{Fe}(\mathrm{L}_{\text{closed}}{}^{\circ})(\mathrm{MeCN})_{2}]{$^{2+}$} & $1 / 2$ & & 0.45 & & -0.17 \\
\hline & $3 / 2$ & & 1.10 & & 2.35 \\
\hline & $5 / 2$ & & 0.95 & & 3.53 \\
\hline
\end{tabular}




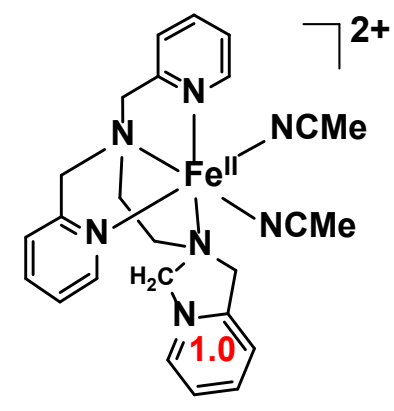

doublet

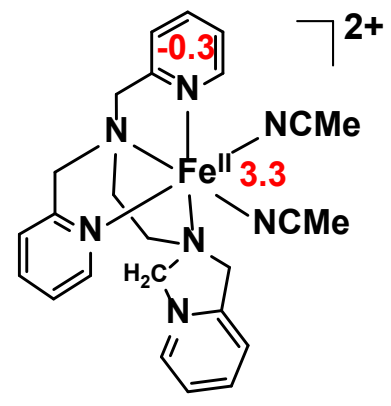

quartet

33.1

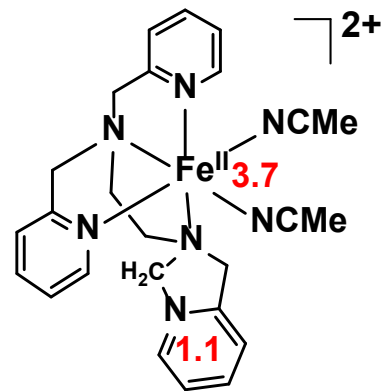

sextet

25.1

Figure S6. Spin density distribution on the $\left[\mathrm{Fe}\left(\mathrm{L}_{\text {closed }}{ }^{\bullet}\right)(\mathrm{MeCN})_{2}\right]^{2+}$ complex in the three spin states considered. 


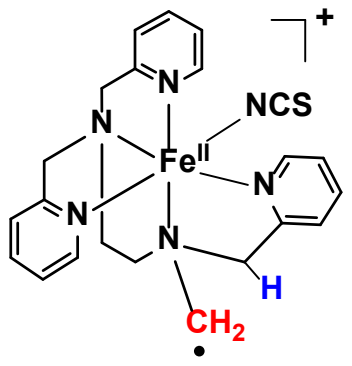

8

doublet

quartet

sextet

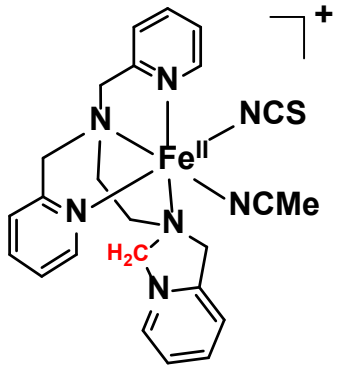

NCMe-NCS

21.4

34.2

13.4

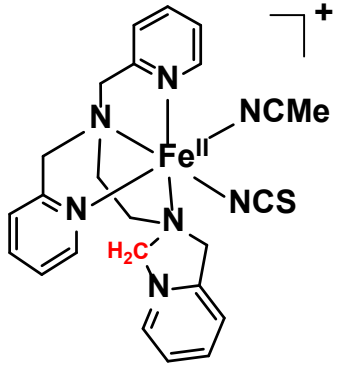

NCS-NCMe

21.4

25.9

12.6

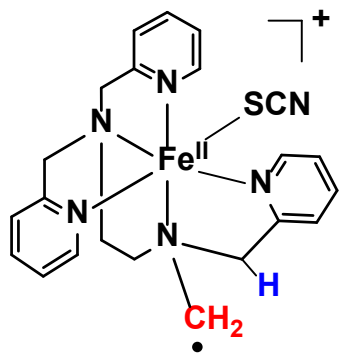

8-SCN

21.7

3.0

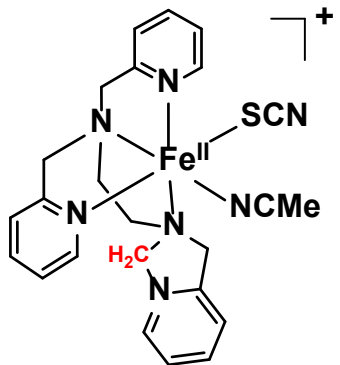

NCMe-SCN

31.2

33.2

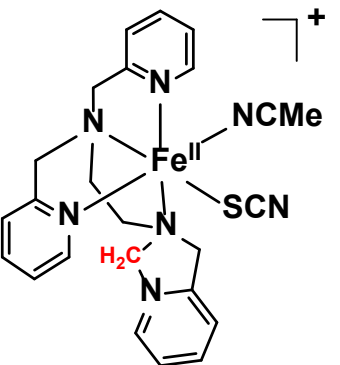

SCN-NCMe

33.3

34.1

18.2

Figure S7. Structures accesible upon irradiation and $\mathrm{CO}_{2}$ release from $[\mathrm{Fe}(\mathrm{S}-\mathrm{SCN})(\mathrm{tpena})]^{+}$. Relative energies are free energies in $\mathrm{kcal} / \mathrm{mol}$ from sextet spin state of the initial complex mer-[Fe ${ }^{\text {III }}$ (tpena) ${ }^{2+}$. Brown: doublet, purple: quartet, black: sextet. No spin distributions are indicated in the 2-D drawings because they differ between spin states. 
Table S10. TD-DFT results on absorption bands for the possible intermediatiens upon irradiation shown in Figure S7.

\begin{tabular}{|c|c|}
\hline 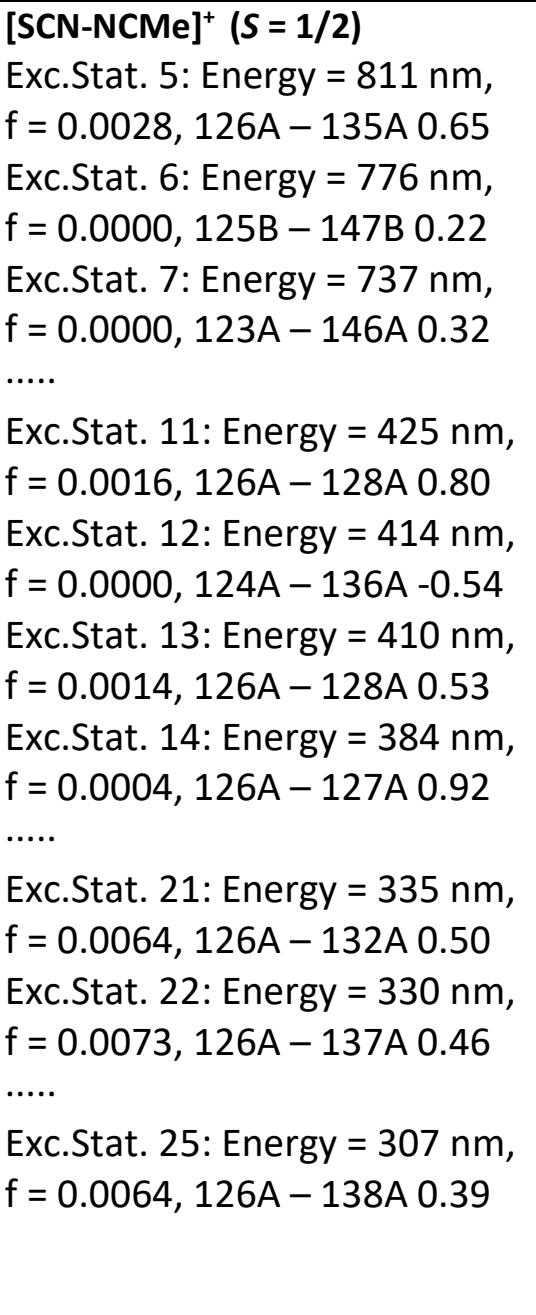 & 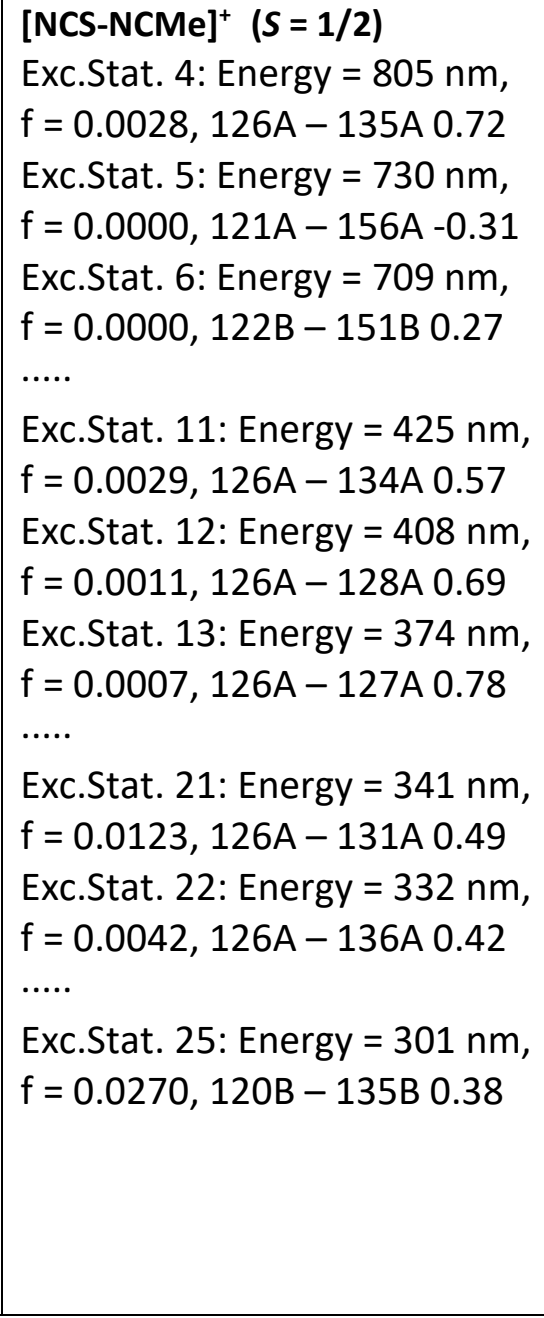 \\
\hline
\end{tabular}




\begin{tabular}{|c|c|}
\hline 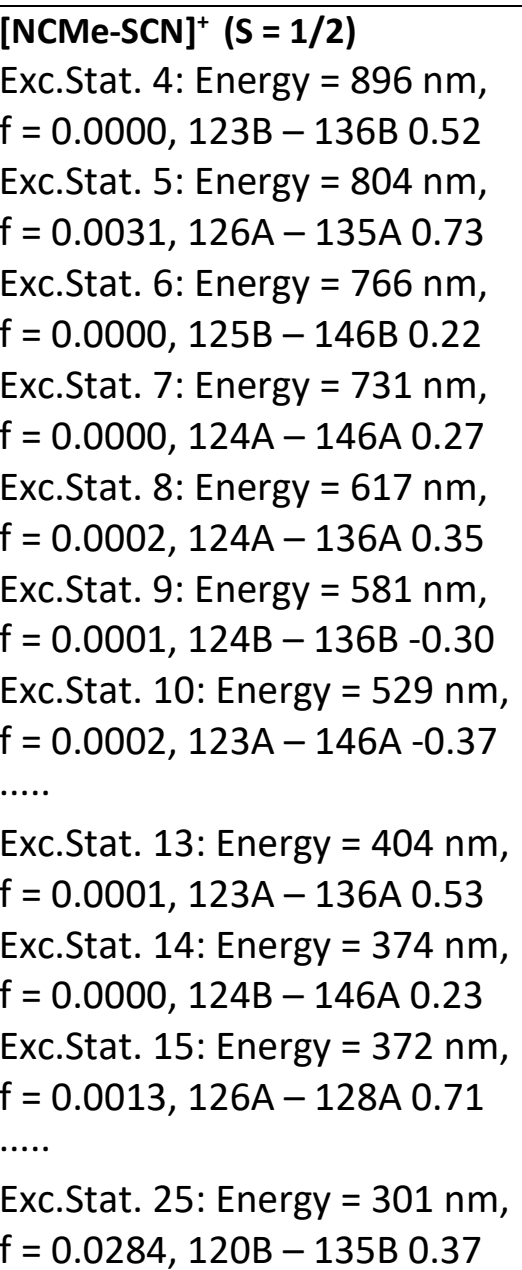 & 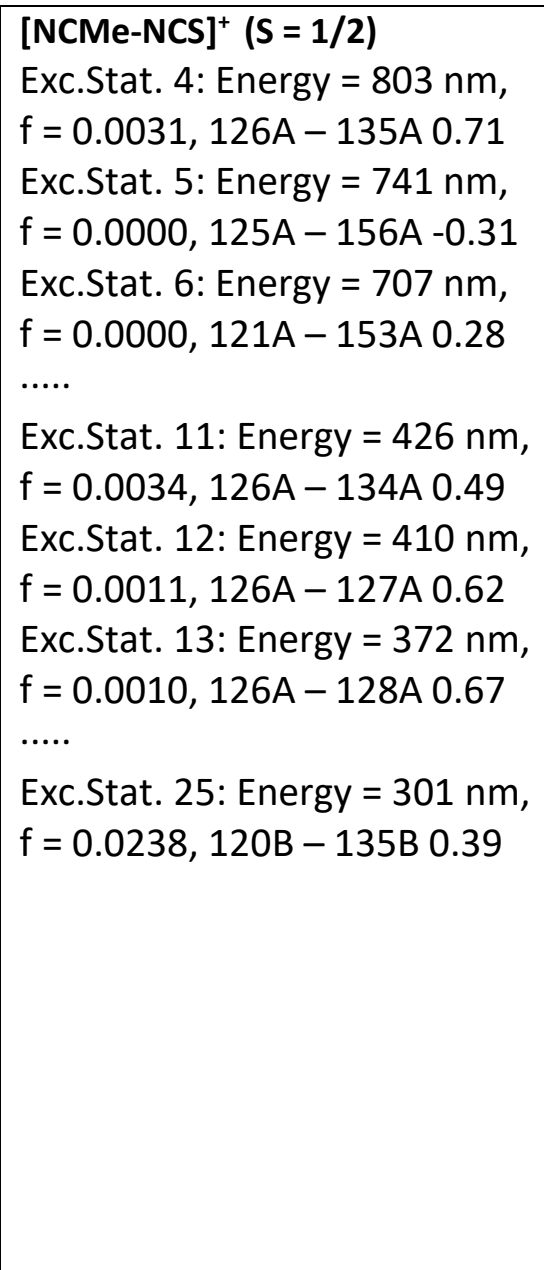 \\
\hline
\end{tabular}




\begin{tabular}{|c|c|}
\hline 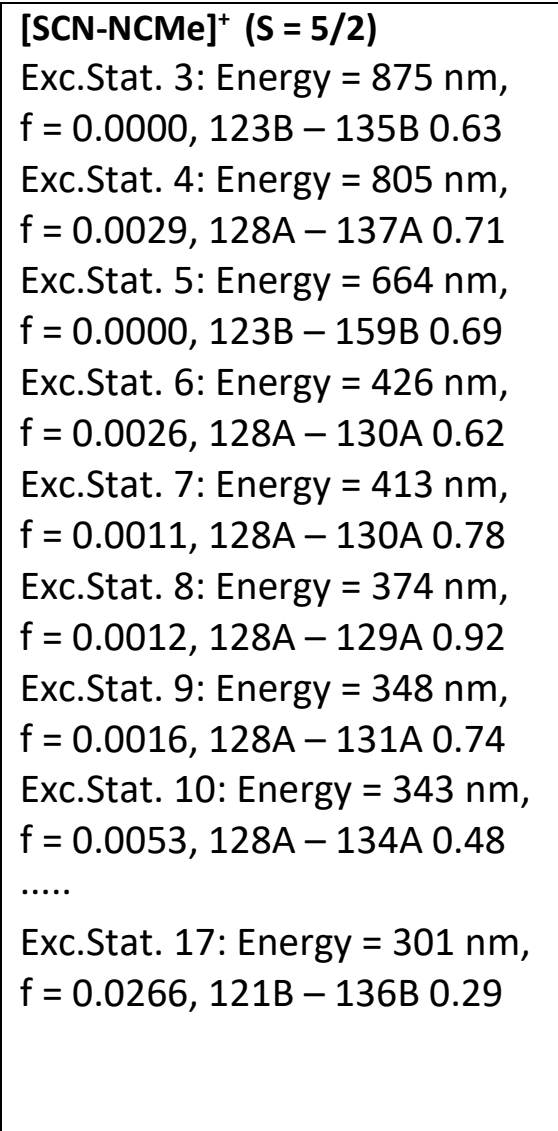 & 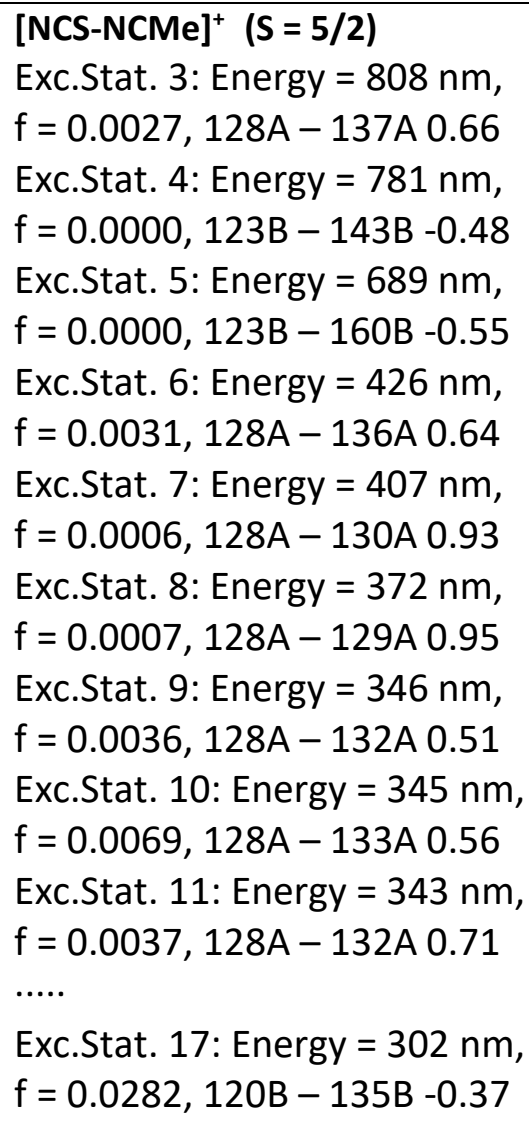 \\
\hline
\end{tabular}




\begin{tabular}{|c|c|}
\hline 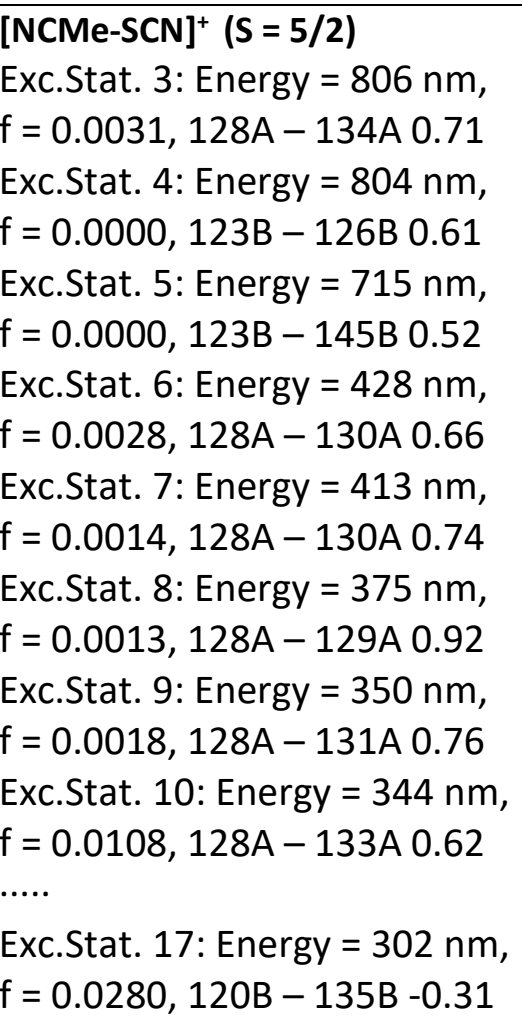 & 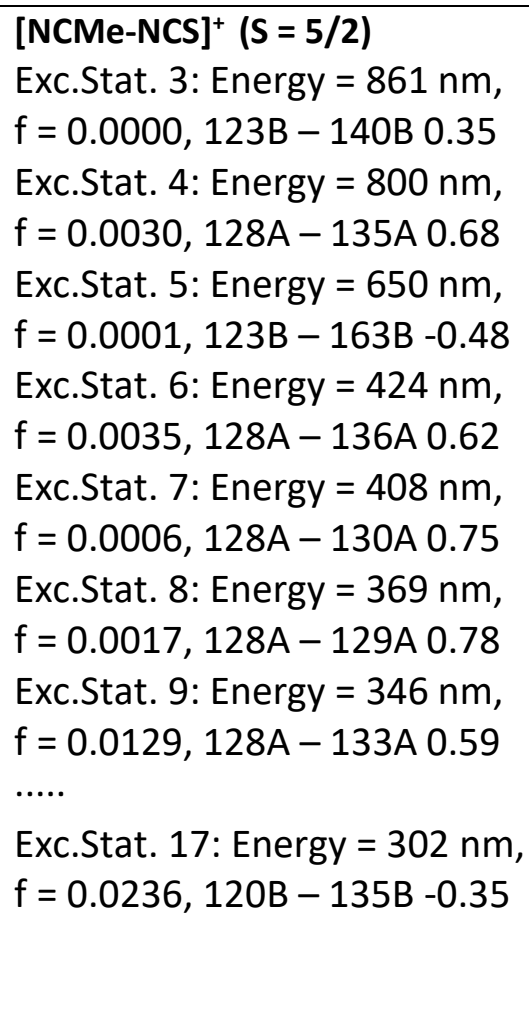 \\
\hline
\end{tabular}

\begin{tabular}{|c|c|}
\hline 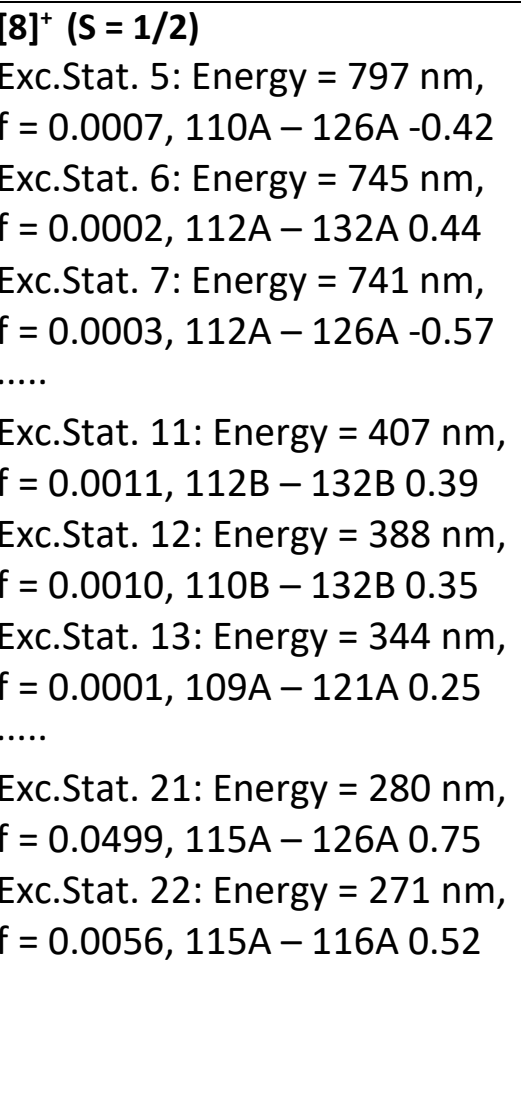 & 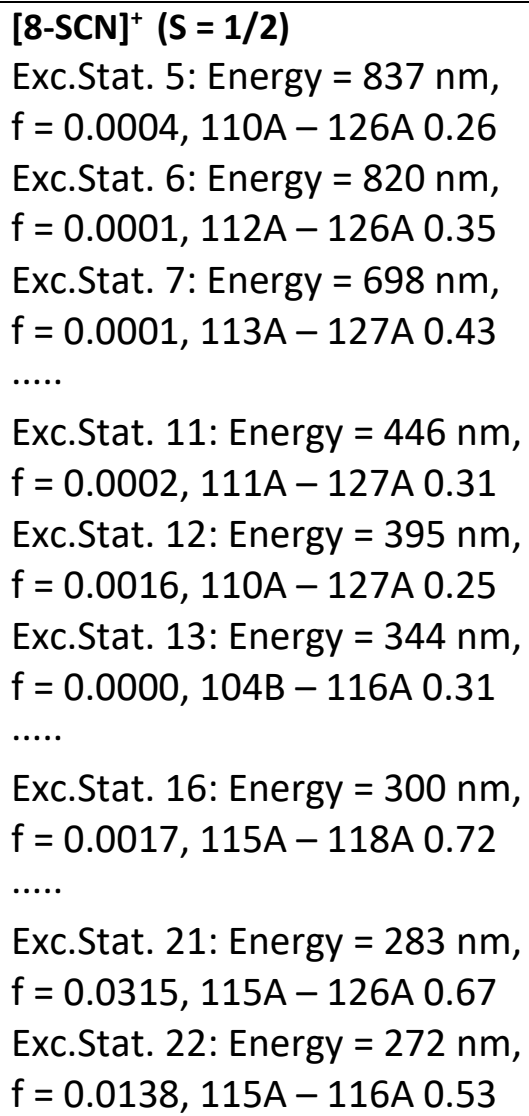 \\
\hline
\end{tabular}




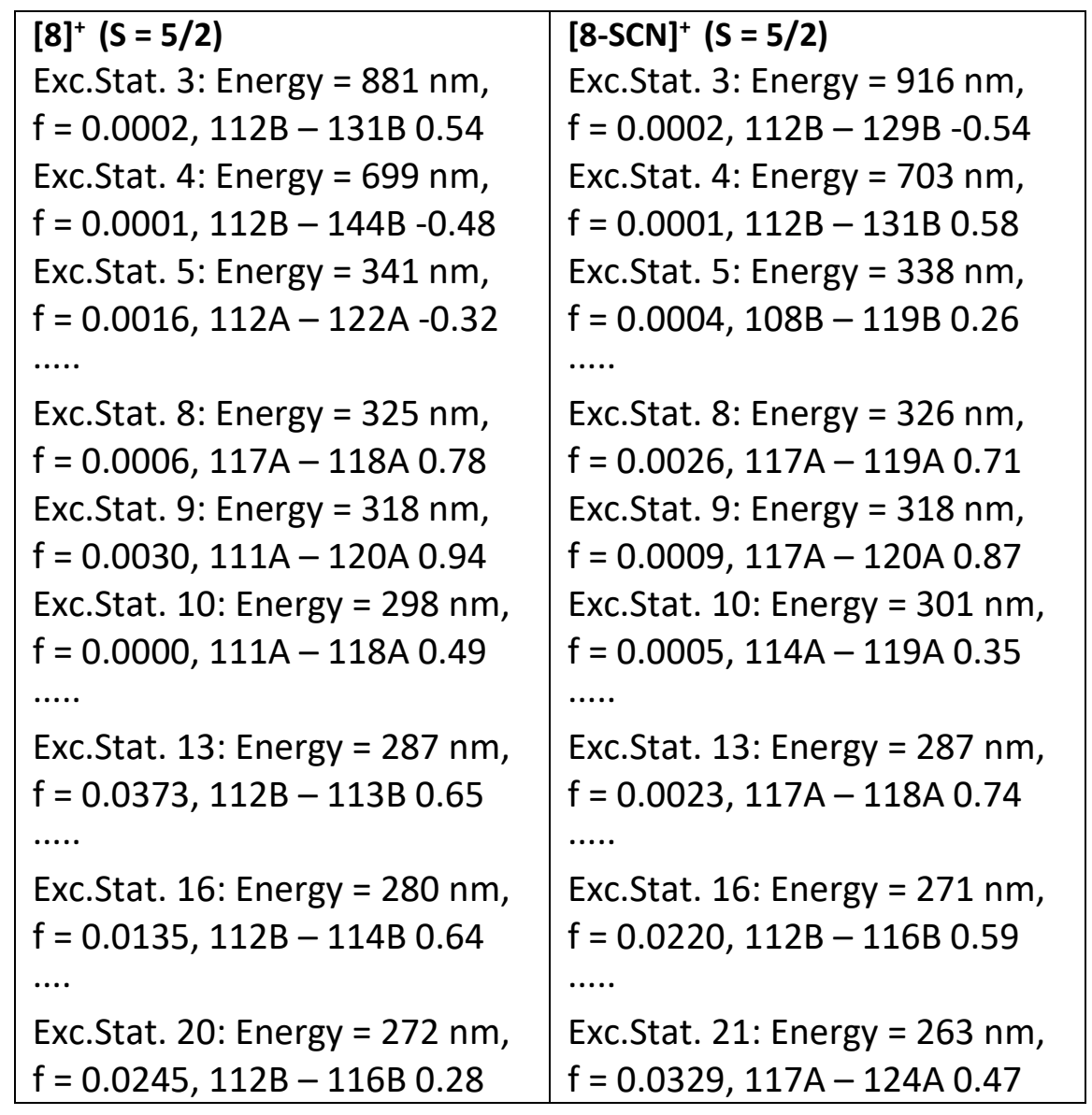

